\title{
Early clinical characteristics of bacterial endophthalmitis in retinopathy of prematurity after intravitreal bevacizumab injection: A case report
}

\author{
JIANXUN WANG and DAOMAN XIANG
}

Department of Ophthalmology, Guangzhou Women and Children Medical Center, Guangzhou, Guangdong 510623, P.R. China

Received February 2, 2016; Accepted February 14, 2017

DOI: $10.3892 /$ etm.2017.4406

\begin{abstract}
Intravitreal bevacizumab injection (IVB) is emerging as a safe and effective therapy for retinopathy of prematurity (ROP); however, follow-up investigations after IVB have indicated that endophthalmitis, a rare and devastating complication, may develop. The present study reports a case of an infant with ROP who developed endophthalmitis after IVB infection. The infant was administered with an intravitreal injection of broad-spectrum antibiotics to treat the endophthalmitis. A favorable anatomic outcome was achieved after follow-up. From this case, it was discovered that the early clinical characteristics of endophthalmitis infection secondary to IVB in premature infants include: i) The clinical symptoms of endophthalmitis infection appearing as early as 3-5 days after IVB; ii) a white membranous plaque on the retina surface with an obscure circular boundary; iii) a plaque that enlarges to a gray flocculent mass and intrudes the vitreous body, where the hazing surrounds the lesion; and iv) conjunctival hyperemia and cornea edema in the anterior segment of the affected eye. This case also indicated that timely retinal screening with RetCam or a binocular ophthalmoscope 3-5 days after IVB may be effective for detecting early stage endophthalmitis in infants who are unable to complain of a loss of visual acuity. From the present case report, it is advisable that the same type of screening be performed 3-5 days after other types of eye surgery, such as cataract extraction surgery, to detect early stage endophthalmitis. Furthermore, the present case also revealed that an early intravitreal injection of broad-spectrum antibiotics may be an effective treatment for premature infants with bacterial endophthalmitis.
\end{abstract}

Correspondence to: Dr Daoman Xiang, Department of Ophthalmology, Guangzhou Women and Children Medical Center, 8 Jinsui Road, Guangzhou, Guangdong 510623, P.R. China E-mail: xiangdm35@126.com

Key words: retinopathy of prematurity, endophthalmitis, intravitreal injection, bevacizumab, antibiotics, infants

\section{Introduction}

Retinopathy of prematurity (ROP) is an eye disease that is caused by abnormal blood vessel development in premature infants, leading to potential vision loss. It is a major cause of childhood blindness, both in developed and developing countries (1). Prevalence of ROP varies among different countries and the incidence of blindness in infants due to ROP is $\sim 1$ case in 820 infants in the United States (2). ROP is diagnosed using RetCam imaging or a binocular ophthalmoscope. Peripheral retinal ablation with confluent laser therapy is the conventional treatment for type I pre-threshold or threshold ROP; however, this approach is invasive, destructive and is not completely effective against blindness in infants. In contrast, anti-vascular endothelial growth factor (VEGF) drugs have emerged as an alternative therapy with positive outcomes (3-7). Intravitreal injection of bevacizumab (IVB; Avastin ${ }^{\circledR}$ ), a humanized anti-VEGF monoclonal antibody, is an effective and safe therapy for ROP with no major side effects (3-7). IVB is commonly used to treat diseases, including choroidal neovascularization, proliferative diabetic retinopathy and cystoid macular edema due to retinal vein occlusions (8). In adults, intravitreal injections are safe, with few complications, including subconjunctival hemorrhage and corneal abrasion (9). One rare yet harmful complication of intravitreal injections is endophthalmitis, which occurs at a rate of 0.019 (10) to $0.54 \%$ (11). IVB has been approved as a monotherapy or an adjunctive treatment for ROP (3-7). The present case is the first to report bacterial endophthalmitis in an infant with ROP after treatment with IVB.

\section{Case report}

The present case report was conducted at Guangzhou Women and Children's Medical Center (Guangzhou, China). The study was approved by the Guangzhou Women and Children's Medical Center Review and Ethics Board. A male neonate with a gestational age of 28 weeks and a birth weight of $1.2 \mathrm{~kg}$ was admitted to the neonatal intensive care unit of the Guangzhou Women and Children Medical Center on October 2013. A routine retinal screening at 32-weeks postmenstrual age indicated a line between the vascular and avascular areas of the peripheral retina. Two weeks later, retinal disease progressed with the retinal image demonstrating a ridge with dilated 
veins and tortuous arteries in the posterior retina. Using the revised International Classification for ROP (12), an ophthalmologist diagnosed the neonate with stage 2 ROP, zone II and plus disease. The neonate had pneumonia and received prolonged supplemental oxygen; therefore, considering the risk of general anesthesia when performing laser photocoagulation, we chose IVB for ROP treatment instead of laser photocoagulation.

The parents of the patient were informed of the side effects of IVB treatment and the follow-up visits that would be required after treatment. Written informed consent was obtained from the parents prior to surgery. The injection was administered in an operating room under the care of an experienced pediatric anesthesiologist. A topical anesthetic, $4 \%$ oxybuprocaine hydrochloride in the form of eye drops (Benoxil; Santen Pharmaceutical Co., Ltd., Osaka, Japan), was applied to both eyes and a $0.1 \%$ povidone iodine solution (PVP-I; Adf, Ltd., Guangzhou, China) was applied for surgical asepsis. Bevacizumab $(0.625 \mathrm{mg}$ in $0.25 \mathrm{ml})$ was then injected $1 \mathrm{~mm}$ posterior to the limbus, initially perpendicular to the globe and then towards the center. After surgery, tobramycin/dexamethasone eye ointments (Tobradex; Alcon Laboratories, Fort Worth, TX, USA) were applied topically three times daily. No systemic antibiotic was used.

Four days after surgery, retinal imaging using RetCam (Massie Research Laboratories, Inc., Dublin, CA, USA) demonstrated a decrease in the size of the ridge and plus disease in both eyes. However, a circular membranous white plaque with an obscure boundary was observed on the retinal surface of the left eye (Fig. 1). There was no neovascularization or fibroplasia adjacent to the lesion. Neither hypopyon nor conjunctival hyperemia were observed using a portable slit lamp microscope.

Three days following the first follow-up, retinal imaging demonstrated that the plaque had enlarged to a gray flocculent mass that intruded into the vitreous body, where hazing surrounded the lesion (Fig. 2). A diagnosis of bacterial endophthalmitis in the left eye was made based on the characteristics of the lesion and the rapid deterioration. Conjunctival hyperemia and cornea edema were observed later on the same day. A vitreous tap procedure was immediately performed using a trans-pars plana vitreous 29-gauge needle to collect a $0.05-\mathrm{ml}$ vitreous sample. Following this, an intravitreal injection of vancomycin $(0.3 \mathrm{mg}$ in $0.03 \mathrm{ml}$; Vianex S.A., Pallini Attiki, Greece) plus ceftazidime $(0.75 \mathrm{mg}$ in $0.03 \mathrm{ml}$; Zhijun Pharmaceutical Co., Ltd., Shenzhen, China) was administered. One drop of tobramycin/dexamethasone was applied to the eye every $3 \mathrm{~h}$ for 3 days, followed by the application of tobramycin/dexamethasone ointments three times per day for 1 week. No systemic antibiotics were used.

Cultures of the vitreous biopsy material and blood were negative for microorganisms, including bacteria and fungi. Four days after intravitreal injection of antibiotics, conjunctival hyperemia, cornea edema and the retinal lesion were diminished. After two follow-up examinations that were performed 4 days and 2 weeks after the intravitreal injection, the disease regressed (Figs. 3 and 4, respectively). A favorable anatomic outcome was achieved 3 weeks after the intravitreal injection of antibiotics (Fig. 5).

\section{Discussion}

In the present case study, a white membranous plaque with an obscure circular boundary was observed on the retinal surface of the left eye of an infant with ROP four days after IVB. Retinoblastoma was considered as a possible diagnosis based on the appearance of the fundus. Retinoblastoma is a tumor that slowly develops under the retina at an early stage and may be creamy or yellow-white with a yogurt-like appearance (13). However, in the present case, retinal imaging indicated that the plaque enlarged to a gray flocculent mass that intruded into the vitreous body, with hazing surrounding the lesion. Based on the observation of the appearance of the mass, it was concluded that the mass was intraocular inflammation instead of retinoblastoma.

Intravitreal injection may cause infectious or non-infectious intraocular inflammatory reactions and it is critical to determine the type of inflammatory reaction so that the appropriate treatment method is applied. A bevacizumab-induced non-infectious reaction is characterized by early onset and a drop in visual acuity. In addition, patients exhibit a marked anterior chamber reaction without hypopyon. Large cellular aggregates of pseudogranulomatous aspect have also been observed in patients with a bevacizumab-induced non-infectious reaction in the absence of positive microbiological findings (14). No apparent benefits of systemic treatment with antibiotics have been observed in these patients; however, systemic or topical cortisone treatment have been demonstrated to be more effective (14). In the present case, the intraocular inflammatory reaction was more severe than the non-infectious inflammation described above. Conjunctival hyperemia and cornea edema were observed in the infected eye and the retinal lesion became larger and intruded the vitreous body. In addition, the inflammation did not respond to topical steroid treatment. Finally, the infant was treated with IVB in both eyes; however, only the left eye was inflamed, and so the case did not appear to be non-infectious inflammation induced by IVB.

Candida sepsis is a risk factor for ROP in infants (15) and may also cause endogenous fungal endophthalmitis in premature babies (16). The typical manifestations of Candida endophthalmitis include chorioretinitis, vitritis, fluffy vitreous lesions, Roth's spots, uveitis and hypopyon $(16,17)$. A case with bilateral Candida endophthalmitis associated with ROP presented moderate diffuse vitreous consolidation and focal intravitreal inflammatory aggregates (18). In the present case, the lesion progressed quickly, and as there was an obvious intravitreal injection history this suggested that the infection was not endogenous fungal endophthalmitis.

Patients with acute postoperative bacterial endophthalmitis typically experience reduced vision, progressive pain, eyelid edema, conjunctival infection and chemosis within 2 weeks of surgery (19). Bacterial endophthalmitis was diagnosed in the present case based on the intravitreal injection history and clinical signs. Out of 200 bevacizumab injections administered at Guangzhou Women and Children Medical Center from 2010 to 2013 , this was the only case that demonstrated endophthalmitis and is to the best of our knowledge the first case reported in the literature. 


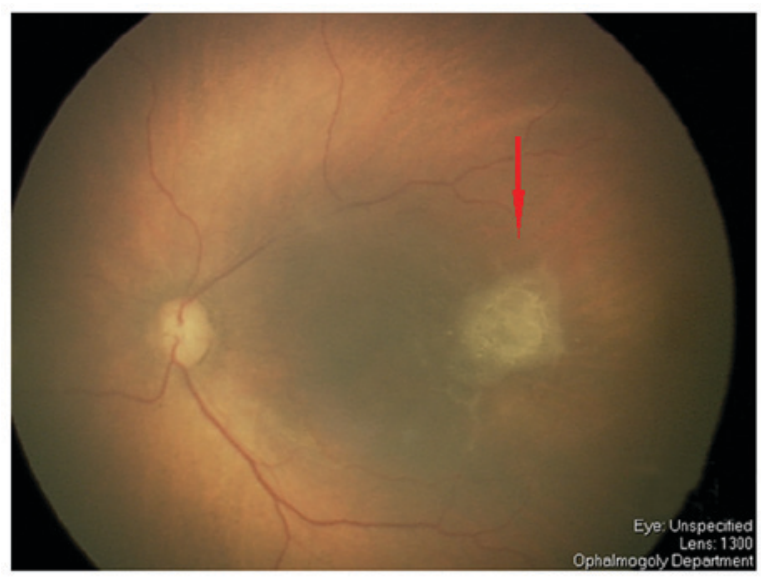

Figure 1. A white lesion on the retina surface of the left eye, indicated by the red arrow, was observed 4 days after intravitreal bevacizumab injection was used to treat retinopathy of prematurity.

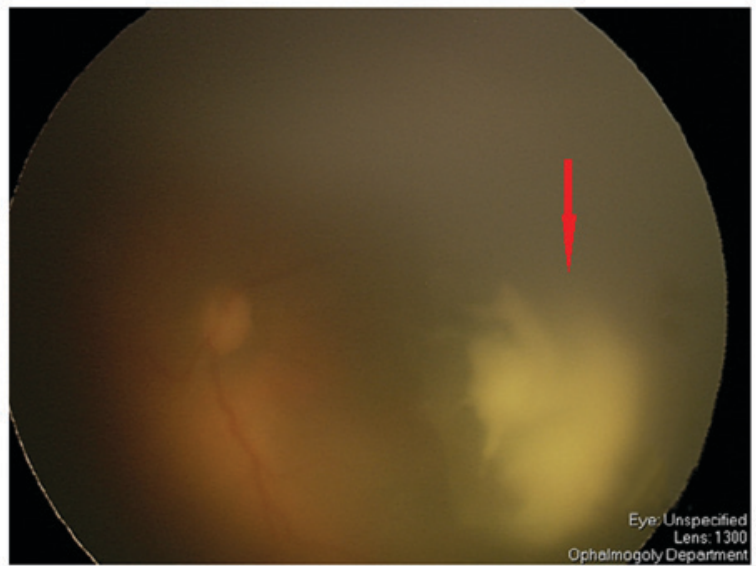

Figure 2. Three days after the first follow-up, the white lesion enlarged to a gray flocculent mass and intruded the vitreous body, as indicated by the red arrow, where hazing was detected. The lesion was diagnosed as bacterial endophthalmitis.

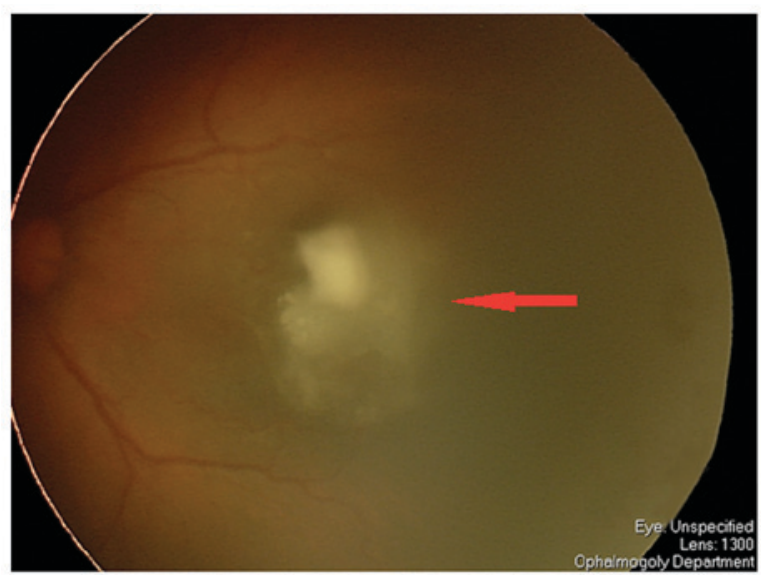

Figure 3. Regression of bacterial endophthalmitis was observed 4 days after intravitreal injection of antibiotics, as indicated by the red arrow.

An intravitreal injection of broad-spectrum antibiotics was administered in the present case as even a slight delay in treatment may result in severe vision loss. The infant

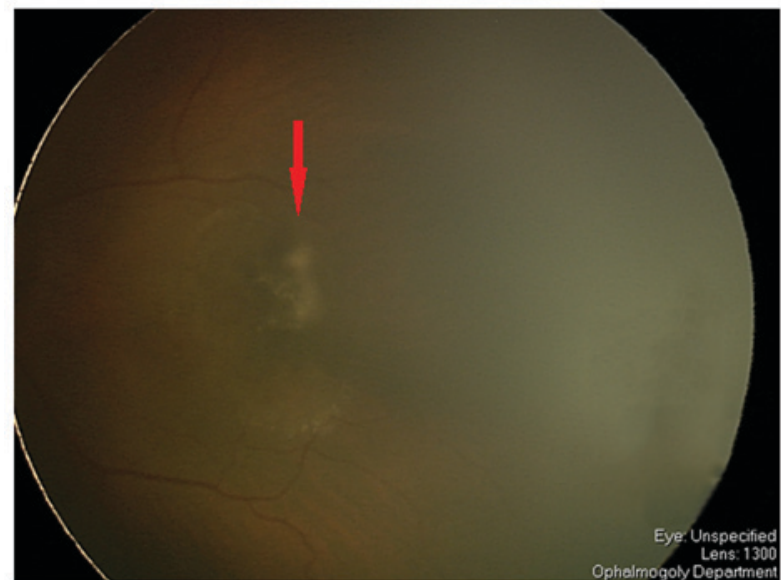

Figure 4. Bacterial endophthalmitis regressed 2 weeks after intravitreal injection of antibiotics, as indicated by the red arrow.

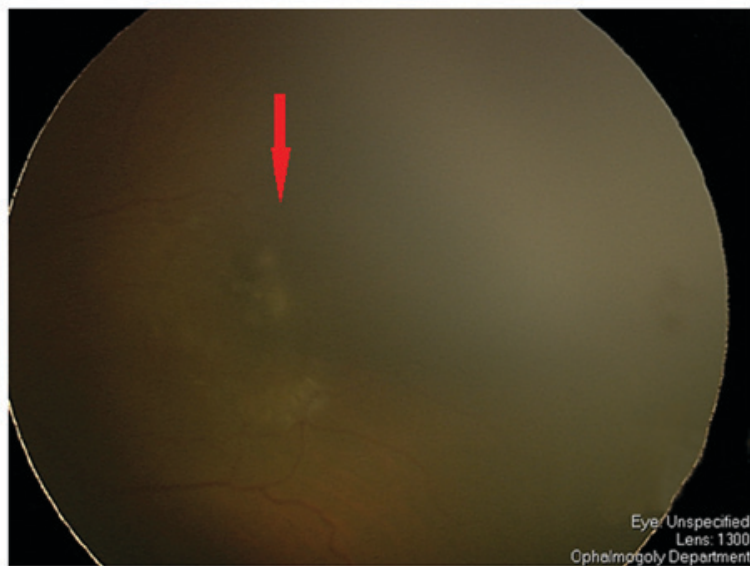

Figure 5. The retina lesion disappeared and a favorable anatomic outcome was achieved 3 weeks after intravitreal injection of antibiotics, as indicated by the red arrow.

responded positively, confirming that the endophthalmitis present was caused by a bacterial infection. In cases involving postoperative bacterial endophthalmitis, only $69 \%$ of cultures demonstrate positive bacterial growth (20). In the present case, the negative results obtained from the vitreous biopsy culture may have resulted from the high density of the infant's vitreous body, rather than the dissemination of the bacteria.

As endophthalmitis is able to severely damage vision, it has been suggested that broad-spectrum antibiotics should be used prior to the differentiation of the infectious organisms. Ceftazidime plus vancomycin may be effective antibiotics to treat endophthalmitis (21). The established intravitreal injection dosages for adults are $2.25 \mathrm{mg} / 0.1 \mathrm{ml}$ ceftazidime and $1.0 \mathrm{mg} / 0.1 \mathrm{ml}$ vancomycin. However, the optimal or recommended dosages for infants are unknown. The present study used one-third of the adult dosage $(0.06 \mathrm{ml})$ with the same concentration. This dosage was based on the approximation that a premature infant's eye is one-third of the volume of an adult eye, and the reported maximal volume for IVB in premature infants without complications is $0.05 \mathrm{ml}(1.25 \mathrm{mg})(22)$. Fortunately, in the present case, the bacterial infection was controlled without complications. After several follow-up 
visits, a favorable anatomic outcome was achieved. This result confirmed that the white membranous plaque with an obscure circular boundary identified 4 days after IVB was a bacterial endophthalmitis infection.

In conclusion, the present case highlighted the early clinical characteristics of endophthalmitis infection secondary to IVB in premature infants. These clinical characteristics include: i) Detection of clinical indicators at 3-5 days after IVB; ii) a white membranous plaque on the retina surface with an obscure circular boundary; iii) a plaque that enlarges to a gray flocculent mass and intrudes the vitreous body, where hazing surrounds the lesion; and iv) conjunctival hyperemia and cornea edema in the anterior segment of the affected eye. This case also indicated that timely retinal screening with RetCam or a binocular ophthalmoscope 3-5 days after IVB may be effective for detecting early stage endophthalmitis in infants who are unable to complain of a loss of visual acuity. The same type of screening should be performed 3-5 days after other types of eye surgery, such as cataract extraction surgery, to detect early stage endophthalmitis. Furthermore, the present case also revealed that an early intravitreal injection of broad-spectrum antibiotics may be an effective treatment for premature infants with bacterial endophthalmitis.

\section{Acknowledgements}

The collection and writing of the present research was supported by the Major Guangzhou Science and Technology Plan Project 2012 (grant no. 2012Y2-00017) and Guangdong Province Nature Science Fund of China (grant no. S2011010004440).

\section{References}

1. Gilbert C: Retinopathy of prematurity: A global perspective of the epidemics, population of babies at risk and implications for control. Early Hum Dev 84: 77-82, 2008.

2. LadEM,Nguyen TC,Morton JM and Moshfeghi DM: Retinopathy of prematurity in the United States. Br J Ophthalmol 92: 320-325, 2008.

3. Chung EJ, Kim JH, Ahn HS and Koh HJ: Combination of laser photocoagulation and intravitreal bevacizumab (Avastin) for aggressive zone I retinopathy of prematurity. Graefes Arch Clin Exp Ophthalmol 245: 1727-1730, 2007.

4. Mintz-Hittner HA and Kuffel RR Jr: Intravitreal injection of bevacizumab (avastin) for treatment of stage 3 retinopathy of prematurity in zone I or posterior zone II. Retina 28: 831-838, 2008.

5. Quiroz-Mercado H, Martinez-Castellanos MA, HernandezRojas ML, Salazar-Teran N and Chan RV: Antiangiogenic therapy with intravitreal bevacizumab for retinopathy of prematurity. Retina 28 (3 Suppl): S19-S25, 2008.
6. Travassos A, Teixeira S, Ferreira P, Regadas I, Travassos AS, Esperancinha FE, Prieto I, Pires G, van Velze R, Valido A and Machado Mdo C: Intravitreal bevacizumab in aggressive posterior retinopathy of prematurity. Ophthalmic Surg Lasers Imaging 38: 233-237, 2007.

7. Mintz-Hittner HA, Kennedy KA and Chuang AZ; BEAT-ROP Cooperative Group: Efficacy of intravitreal bevacizumab for stage 3+ retinopathy of prematurity. N Engl J Med 364: 603-615, 2011.

8. Spaide RF, Laud K, Fine HF, Klancnik JM Jr, Meyerle CB, Yannuzzi LA, Sorenson J, Slakter J, Fisher YL and Cooney MJ: Intravitreal bevacizumab treatment of choroidal neovascularization secondary to age-related macular degeneration. Retina 26: 383-390, 2006

9. Jager RD, Aiello LP, Patel SC and Cunningham ET Jr: Risks of intravitreous injection: A comprehensive review. Retina 24: 676-698, 2004.

10. Mason JO III, White MF, Feist RM, Thomley ML, Albert MA, Persaud TO, Yunker JJ and Vail RS: Incidence of acute onset endophthalmitis following intravitreal bevacizumab (Avastin) injection. Retina 28: 564-567, 2008.

11. Diabetic Retinopathy Clinical Research Network, Scott IU, Edwards AR, Beck RW, Bressler NM, Chan CK, Elman MJ, Friedman SM, Greven CM, Maturi RK, et al: A phase II randomized clinical trial of intravitreal bevacizumab for diabetic macular edema. Ophthalmology 114: 1860-1867, 2007.

12. International Committee for the Classification of Retinopathy of Prematurity: The international classification of retinopathy of prematurity revisited. Arch Ophthalmol 123: 991-999, 2005.

13. Abramson DH, Beaverson K, Sangani P, Vora RA, Lee TC, Hochberg HM, Kirszrot J and Ranjithan M: Screening for retinoblastoma: Presenting signs as prognosticators of patient and ocular survival. Pediatrics 112: 1248-1255, 2003.

14. Sato T, Emi K, Ikeda T, Bando H, Sato S, Morita S, Oyagi T and Sawada K: Severe intraocular inflammation after intravitreal injection of bevacizumab. Ophthalmology 117: 512-516, 516. e1-e2, 2010

15. Mittal M, Dhanireddy R and Higgins RD: Candida sepsis and association with retinopathy of prematurity. Pediatrics 101: 654-657, 1998.

16. Annable WL, Kachmer ML, DiMarco M and DeSantis D: Long-term follow-up of Candida endophthalmitis in the premature infant. J Pediatr Ophthalmol Strabismus 27: 103-106, 1990.

17. Clinch TE, Duker JS, Eagle RC Jr, Calhoun JH, Augsburger JJ and Fischer DH: Infantile endogenous Candida endophthalmitis presenting as a cataract. Surv Ophthalmol 34: 107-112, 1989.

18. Gago LC, Capone A Jr and Trese MT: Bilateral presumed endogenous candida endophthalmitis and stage 3 retinopathy of prematurity. Am J Ophthalmol 134: 611-613, 2002.

19. Mamalis N, Kearsley L and Brinton E: Postoperative endophthalmitis. Curr Opin Ophthalmol 13: 14-18, 2002.

20. No authors listed: Results of the Endophthalmitis Vitrectomy Study. A randomized trial of immediate vitrectomy and of intravenous antibiotics for the treatment of postoperative bacterial endophthalmitis. Endophthalmitis Vitrectomy Study Group. Arch Ophthalmol 113: 1479-1496, 1995.

21. Gentile RC, Shukla S, Shah M, Ritterband DC, Engelbert M, Davis A and $\mathrm{Hu}$ DN: Microbiological spectrum and antibiotic sensitivity in endophthalmitis: A 25-year review. Ophthalmology 121: 1634-1642, 2014.

22. Lalwani GA, Berrocal AM, Murray TG, Buch M, Cardone S, Hess D, Johnson RA and Puliafito CA: Off-label use of intravitreal bevacizumab (Avastin) for salvage treatment in progressive threshold retinopathy of prematurity. Retina 28 (3 Suppl): S13-S18, 2008. 